\title{
Prompt emission of GRB 121217A from gamma-rays to the near-infrared ${ }^{\star}$
}

\author{
J. Elliott ${ }^{1}$, H. -F. Yu ${ }^{1}$, S. Schmidl ${ }^{2}$, J. Greiner ${ }^{1}$, D. Gruber ${ }^{1}$, S. Oates ${ }^{3}$, S. Kobayashi ${ }^{4}$, B. Zhang ${ }^{5}$, J. R. Cummings ${ }^{6,7}$, \\ R. Filgas ${ }^{8}$, N. Gehrels ${ }^{9}$, D. Grupe ${ }^{10}$, D. A. Kann ${ }^{1}$, S. Klose ${ }^{2}$, T. Krühler ${ }^{11,12}$, A. Nicuesa Guelbenzu ${ }^{2}$, A. Rau ${ }^{1}$, \\ A. Rossi ${ }^{2}$, M. Siegel ${ }^{10}$, P. Schady ${ }^{1}$, V. Sudilovsky ${ }^{1}$, M. Tanga ${ }^{1}$, and K. Varela ${ }^{1}$
}

1 Max-Planck-Institut für extraterrestrische Physik, Giessenbachstraße 1, 85748 Garching, Germany e-mail: jonnyelliott@mpe.mpg.de

2 Thüringer Landessternwarte Tautenburg, Sternwarte 5, 07778 Tautenburg, Germany

3 Mullard Space Science Laboratory, University College London, Holmbury St. Mary, Dorking Surrey, RH5 6NT, UK

4 Astrophysics Research Institute, Liverpool John Moores University, IC2, Liverpool Science Park, 146 Brownlow Hill, Liverpool L3 5RF, UK

5 Department of Physics and Astronomy, University of Nevada, Las Vegas, NV89154, USA

${ }^{6}$ University of Maryland Baltimore County, 1000 Hilltop Circle, Baltimore, MD 21250, USA

7 Center for Research and Exploration in Space Sciences and Technology, NASA Goddard Space Flight Center, Greenbelt, MD 20771, USA

${ }^{8}$ Institute of Experimental and Applied Physics, Czech Technical University in Prague, Horská 3a/22, 12800 Prague, Czech Republic

9 Astrophysics Science Division, NASA, Goddard Space Flight Center, Greenbelt, MD 20771, USA

10 Department of Astronomy and Astrophysics, Pennsylvania State University, 525 Davey Laboratory, University Park, PA 16802 , USA

11 Dark Cosmology Centre, Niels Bohr Institute, University of Copenhagen, Juliane Maries Vej 30, 2100 København Ø, Denmark

12 European Southern Observatory, Alonso de Córdova 3107, Vitacura, 19001 Casilla, Santiago 19, Chile

Received 3 September 2013 / Accepted 13 December 2013

\section{ABSTRACT}

\begin{abstract}
The mechanism that causes the prompt-emission episode of gamma-ray bursts (GRBs) is still widely debated despite there being thousands of prompt detections. The favoured internal shock model relates this emission to synchrotron radiation. However, it does not always explain the spectral indices of the shape of the spectrum, which is often fit with empirical functions, such as the Band function. Multi-wavelength observations are therefore required to help investigate the possible underlying mechanisms that causes the prompt emission. We present GRB 121217A, for which we were able to observe its near-infrared (NIR) emission during a secondary prompt-emission episode with the Gamma-Ray burst Optical Near-infrared Detector (GROND) in combination with the Swift and Fermi satellites, which cover an energy range of 5 orders of magnitude $\left(10^{-3} \mathrm{keV}\right.$ to $\left.100 \mathrm{keV}\right)$. We determine a photometric redshift of $z=3.1 \pm 0.1$ with a line-of-sight with little or no extinction $\left(A_{V} \sim 0 \mathrm{mag}\right)$ utilising the optical/NIR SED. From the afterglow, we determine a bulk Lorentz factor of $\Gamma_{0} \sim 250$ and an emission radius of $R<10^{18} \mathrm{~cm}$. The prompt-emission broadband spectral energy distribution is well fit with a broken power law with $\beta_{1}=-0.3 \pm 0.1$ and $\beta_{2}=0.6 \pm 0.1$ that has a break at $E=6.6 \pm 0.9 \mathrm{keV}$, which can be interpreted as the maximum injection frequency. Self-absorption by the electron population below energies of $E_{\mathrm{a}}<6 \mathrm{keV}$ suggest a magnetic field strength of $B \sim 10^{5} \mathrm{G}$. However, all the best fit models underpredict the flux observed in the NIR wavelengths, which also only rebrightens by a factor of $\sim 2$ during the second prompt emission episode, in stark contrast to the $\mathrm{X}$-ray emission, which rebrightens by a factor of $\sim 100$. This suggests an afterglow component is dominating the emission. We present GRB 121217A, one of the few GRBs that has multi-wavelength observations of the prompt-emission period and shows that it can be understood with a synchrotron radiation model. However, due to the complexity of the GRB's emission, other mechanisms that result in Band-like spectra cannot be ruled out.
\end{abstract}

Key words. gamma-ray burst: general - gamma-ray burst: individual: GRB 121217A - X-rays: individuals: GRB 121217A

\section{Introduction}

Ever since gamma-ray bursts (GRBs) were first detected in the 1960s (Klebesadel et al. 1973), satellites have been launched to expand our understanding of the underlying mechanism that causes them. The most notable are the instrument BATSE (Fishman et al. 1989) onboard the CGRO satellite, the Swift (Gehrels et al. 2004) satellite, and Fermi (Atwood et al. 2009) satellite, which were launched during 1990-2008. They have collectively detected thousands of long-duration GRBs and

\footnotetext{
$\star$ Appendix A is available in electronic form at http://www. aanda.org
}

acquired many prompt-emission light curves and gamma-ray spectra. Even though this huge data set has answered many questions about the GRB phenomenon, the underlying problem of the prompt-emission mechanism remains elusive (for a review, see Zhang 2011, 2012).

The standard model of a long-duration GRB involves a compact object, formed by the collapse of a massive rapidly rotating star (Woosley 1993; Paczynski 1998; MacFadyen \& Woosley 1999), that emits jetted relativistic fireballs with different Lorentz factors (Eichler et al. 1989; Narayan et al. 1992; Mészáros 2002). The most commonly discussed model of the prompt emission is the internal-shock scenario (e.g., Rees \& Meszaros 1994), whereby the emitted fireball shells of different 
Lorentz factors cross one another causing relativistic shocks. Fermi acceleration (Fermi 1949) across the shock front in combination with amplified magnetic fields results in the electrons cooling in the form of synchrotron radiation (Sari et al. 1998), primarily at X-ray wavelengths, which is blueshifted into gamma-rays (for a review, see, e.g., Mészáros 2002; Zhang \& Yan 2011). Such a scenario allows easy comparison with observations by fitting power laws to the observed spectra. However, the internal shock model predicts a relatively low radiative efficiency (Kumar 1999; Panaitescu et al. 1999) and a wrong peak energy (unless a small fraction of electrons are accelerated, Daigne \& Mochkovitch 1998). Recent numerical simulations suggest that internal shocks cannot efficiently accelerate particles if the ejecta carries a magnetic field (even if with a moderate magnetisation, Sironi \& Spitkovsky 2009). Also, a fireball giving rise to a strong internal shock emission is expected to have a bright quasi-thermal photosphere component, which is not observed as expected in some GRBs (Zhang \& Pe'er 2009). As a result, alternative models of GRB prompt emission are widely discussed in the literature. These include magnetic dissipation models in a Poynting-flux-dominated flow (e.g., Zhang \& Yan 2011) or a dissipative photosphere model (e.g., Rees \& Mészáros 2005; Vurm et al. 2011). To determine and constrain the preferred mechanism it is crucial to obtain multi-wavelength measurements during prompt-emission episodes of the GRB.

Multi-wavelength measurements of the prompt-emission are not always possible, given the delay between the triggering of gamma-ray telescopes and the slewing of optical instruments. Fortunately, however, there exist tens of fortuitous cases in which both the gamma-ray emission and optical emission have been detected during the prompt period. These can be divided into three possible scenarios: (i) a wide-field camera is observing the same field position as a satellite and so catches the optical emission simultaneously (e.g., 080319B, 130427A; Racusin et al. 2008; Bloom et al. 2009; Beskin et al. 2010; Wren et al. 2013); (ii) the prompt period is long enough that optical instruments slew in time to observe the prompt period (e.g., 990123, 080928, 110205A, 091024; Akerlof et al. 1999; Rossi et al. 2011; Cucchiara et al. 2011; Gruber et al. 2011; Gendre et al. 2012; Zheng et al. 2012; Virgili et al. 2013); and (iii) there is a precursor to the main event so that optical instruments can slew in time (e.g., 041219A, 050820A, 061121; Blake et al. 2005; Vestrand et al. 2005, 2006; Page et al. 2007). Only recently has it become possible to compile samples of bursts that exhibit optical emission during the prompt phase (Kopač et al. 2013). However, their heterogeneous selection means that many more robust detections are required to reach both large number statistics and significant completeness levels.

Despite the successful efforts to detect the optical emission during the prompt episode, there is still no consistent picture on the underlying mechanism (e.g., Kopač et al. 2013). At times, optical emission of GRBs traces the gamma-ray emission, but the optical emission is orders of magnitudes larger than expected by theory (e.g., 110205A). Some do not trace the gamma-ray emission (e.g., 990123), and some are below what is expected (e.g., 061121). A major problem is that the observations are limited to only one filter or are not simultaneous observations, in the majority of cases, which makes it difficult to disentangle temporal and spectral variations. This highlights the importance of simultaneous multi-wavelength observations of the GRB prompt emission to investigate the underlying mechanism.

We add the Swift/Fermi burst GRB 121217A to this handful of cases by first discussing its detections in Sect. 2. Secondly, we present the resulting light curves and spectra in Sect. 3,

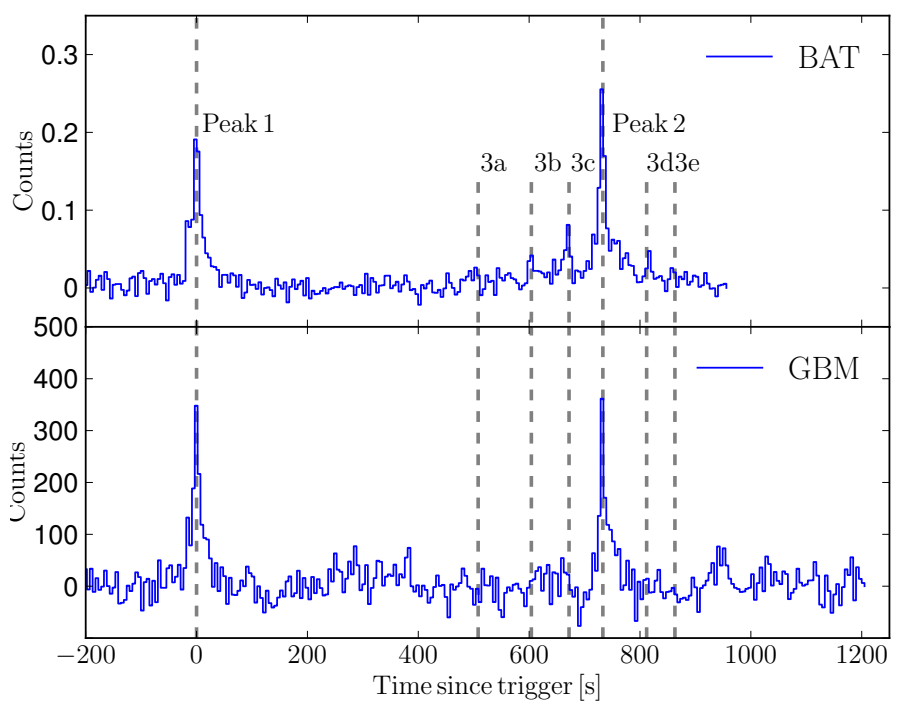

Fig. 1. Gamma-ray light curves of the two prompt episodes of GRB 121217A (Peak 1 and 2) acquired with BAT (15-150 keV) and GBM $(8-1000 \mathrm{keV})$. The GBM triggered on the second peak, which occurs at a time of $T_{0}+735 \mathrm{~s}$, but has been shifted in this plot to coincide with the BAT $T_{0}$. Both light curves have been binned in time with a moving box of $5 \mathrm{~s}$. There is extra emission seen before and after the second peak (3a, 3b, 3c, 3d, and 3e). The corresponding times and instrument detections are noted in Table 1.

discuss the implications in Sect. 4, and then finally conclude in Sect. 5. Throughout, we assume the standard notation of the GRB light curves and spectra of $F(v, t) \propto t^{-\alpha} v^{-\beta}$. In addition, we adopt the notation of $Q_{x}=10^{x} Q$. Unless mentioned otherwise, all uncertainties are quoted to the $1 \sigma$ level. Finally, a $\Lambda$ cold dark matter (CDM) cosmology with the following parameters $\Omega_{\Lambda}=0.7, \Omega_{\mathrm{M}}=0.3$, and $H_{0}=73.0 \mathrm{~km} \mathrm{~s}^{-1} \mathrm{Mpc}^{-1}$ has been used (Freedman \& Madore 2010).

\section{Observations}

\subsection{Swift}

The Burst Alert Telescope (BAT; Barthelmy et al. 2005) mounted on Swift was triggered by GRB 121217A on 17th December 2012 at $T_{0}=07: 17: 47$ UT (Siegel et al. 2012; Cummings et al. 2012). Swift slewed immediately to the burst and the X-Ray Telescope (XRT; Burrows et al. 2005) began observing at $T_{0}+64.0 \mathrm{~s}$ until 15.6 days later (Evans et al. 2012). The BAT light curve was acquired from the Swift quick-look data and the BAT spectrum was acquired from the Swift archive. The prompt light curve can be seen in Fig. 1. The HEAsoft routines batbinevt, bathotpix, batmaskwtevt, batupdatephakw, and batdrmgen were used to generate the BAT PHA and RSP files from the event file in the standard manner. The XRT light curve (Fig. 2) was obtained from the XRT light-curve repository (Evans et al. 2007, 2009) and the XRT spectral data from the public Swift archive. Each spectrum has been regrouped to ensure at least 20 counts per bin using the grppha task from the HEAsoft package using the response matrices from the CALDB v20120209. We assume a Galactic hydrogen column density of $0.4 \times 10^{22} \mathrm{~cm}^{-2}$ (Kalberla et al. 2005).

The prompt emission exhibits two main emission periods separated by a quiescent period of $\sim 500 \mathrm{~s}$ and lasts for a length of $\sim 900 \mathrm{~s}$. There are two main peaks, which are referred to as Peak 1 (first peak) and Peak 2 (second peak). There are also three 
J. Elliott et al.: Prompt emission of GRB 121217A from gamma-rays to the near-infrared

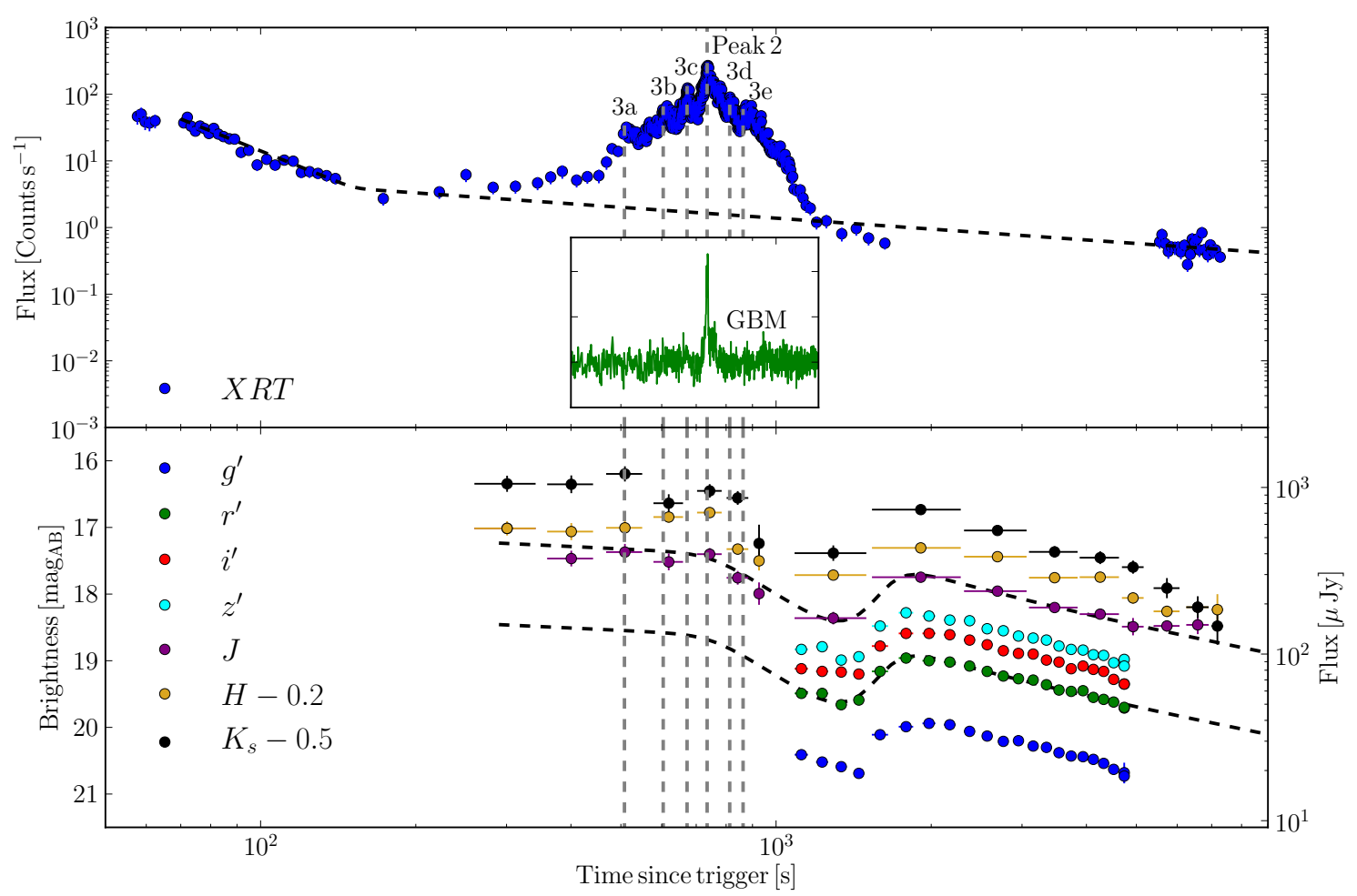

Fig. 2. The X-ray (top) and optical/NIR (bottom) light curves of GRB 121217A with the inset graph showing the GBM prompt emission of the second peak. The canonical model of the X-ray emission can be seen as the black-dashed line in the top panel, where the flare, simultaneous to the second prompt peak, has been excluded from the fit (Sect. 3.3). The black-dashed line in the lower panel is the best-fit double broken power law of the afterglow emission (Sect. 3.4). Only the observations with a time less than $T_{0}+10^{4} \mathrm{~s}$ have been included and the full light curve can be seen in Fig. A.1.

Table 1. Times of gamma-ray emission.

\begin{tabular}{ccccc}
\hline \hline Name & Time $^{a}$ & Duration $^{b}$ & BAT $^{c}$ & $\mathrm{GBM}^{c}$ \\
\hline & $\mathrm{s}$ & $\mathrm{S}$ & & \\
\hline Peak 1 & 0 & 69 & $\mathrm{Y}$ & $\mathrm{Y}$ \\
Peak 2 & 735 & 70 & $\mathrm{Y}$ & $\mathrm{Y}$ \\
3a & 508 & 26 & $\mathrm{Y}$ & $\mathrm{N}$ \\
3b & 604 & 19 & $\mathrm{Y}$ & $\mathrm{Y}^{d}$ \\
3c & 635 & 18 & $\mathrm{Y}$ & $\mathrm{N}$ \\
3d & 813 & 36 & $\mathrm{Y}$ & $\mathrm{N}$ \\
3e & 863 & 27 & $\mathrm{Y}$ & $\mathrm{N}$ \\
\hline
\end{tabular}

Notes. ${ }^{(a)}$ All times are in reference to $T_{0}$ and are taken from BAT. ${ }^{(b)}$ All durations are obtained from BAT and refer to their length, not the $T_{90}$. ${ }^{(c)}$ Detected in this instrument. ${ }^{(d)}$ Excluded due to poor signal-to-noise.

smaller emission peaks prior to the second peak and two after, as depicted in Fig. 1. Their corresponding names and times can be seen in Table 1 .

\section{2. $G B M$}

The Fermi Gamma-ray Burst Monitor (GBM; Atwood et al. 2009) was triggered by Peak 2 on 17th December 2012 at 07:30:02 UT (Yu \& Gruber 2012). Even though GBM's triggering was switched off during the first peak of the prompt emission, as it was moving through a region of high geomagnetic activity, the first peak was still detected. The overall light curve resembles that seen with BAT (Fig. 1). The GBM spectra were reduced in the standard manner using the RMFIT v4.1BA software package and the Response Generator gbmrsp v2.0.
Fluences were determined using CSPEC data (time resolution of $4.096 \mathrm{~s}$ ), and spectral fitting utilised time-tagged event data (time resolution of $64 \mathrm{~ms}$ ).

\subsection{GROND}

The Gamma-Ray burst Optical Near-infrared Detector (GROND; Greiner et al. 2008) at the MPG/ESO $2.2 \mathrm{~m}$ telescope at La Silla, Chile, began observing the field of GRB 121217A at $T=T_{0}+210 \mathrm{~s}$ and located the optical/nearinfrared (NIR) counterpart of GRB 121217A at RA (J2000) = $10^{\mathrm{h}} 14^{\mathrm{m}} 50.4^{\mathrm{s}}$, Dec $(\mathrm{J} 2000)=-62^{\circ} 21^{\prime} 0{ }^{\prime} \cdot 4$ (Elliott et al. 2012b) to an uncertainty of $0{ }^{\prime} 5$. As a result of the XRT position being at the edge of the BAT error circle, the afterglow was outside the field-of-view of the optical detectors for the first $500 \mathrm{~s}$ of observations. Therefore, there is only coverage in the NIR filters due to the larger field-of-view of the NIR detectors. The telescope was then repointed, yielding a short interruption in the observations around $1000 \mathrm{~s}$. The follow-up campaign lasted for 21 days until the afterglow was no longer detected. No underlying candidate host galaxy was discovered to a limit of $r_{\mathrm{AB}}^{\prime}>24.9$ mag.

Image reduction and photometry of the GROND observations were carried out using standard IRAF tasks (Tody 1993) in the way outlined by Krühler et al. (2008) and Yoldaş et al. (2008). In brief, a point-spread function (PSF) was obtained from the bright stars within the field and applied to the afterglow. The absolute calibration of the optical photometry was achieved by observing a Sloan Digital Sky Survey (SDSS) field (Aihara et al. 2011) at RA $(J 2000)=10^{\mathrm{h}} 50^{\mathrm{m}} 36.0^{\mathrm{s}}$, Dec $(\mathrm{J} 2000)=$ $-21^{\circ} 36^{\prime} 00^{\prime \prime}$ and the GRB field consecutively under photometric 
conditions. The NIR absolute calibration was obtained from the Two Micron Sky Survey (2MASS) stars (Skrutskie et al. 2006) within the field of the GRB. The magnitudes are corrected for a Galactic dust reddening of $E_{B-V}^{\mathrm{Gal}}=0.324 \mathrm{mag}$ corresponding to an extinction of $A_{V}^{\mathrm{Gal}}=1.0 \mathrm{mag}$ for $R_{V}=3.1$ (Schlafly \& Finkbeiner 2011). The magnitudes of GRB 121217A and its reference stars can be found in Tables A.1 to A.3.

\section{Results}

\subsection{Redshift}

A spectral energy distribution was constructed from the GROND filters at a mid-time of $T_{0}+31.4 \mathrm{~min}$, when the optical counterpart is the afterglow component (see Sect. 3.4 for more details). Within the framework of the standard fireball model, external shocks emit synchrotron radiation, which is described by a (broken) power law (e.g., Sari et al. 1998). These power laws are then modified by the GRB host's intrinsic extinction and the GRBs redshift, which determines the position of the Lyman-break. To find the redshift and intrinsic host dust extinction, we followed the prescription outlined in Krühler et al. (2011) and fit power laws over a grid of parameters consisting of: spectral slope $\beta=0.01-2.00$ in steps of 0.01 , host dust $A_{V}=0.0-0.5$ mag in steps of 0.02, dust models (Milky Way, MW; Large Magellanic Cloud, LMC; and Small Magellanic Cloud, SMC), and redshift $z=0.0-5.0$ in steps of 0.06 . The best fit solution is determined from the minimum $\chi^{2}$ value and the uncertainties from the corresponding $\chi^{2}$ contours. We find a best fit solution with $\chi^{2}=3.7$ and 3 degrees of freedom (d.o.f.) for the SMC-like dust model, $\beta=0.87_{-0.07}^{+0.04}, A_{V}=0.00_{-0.00}^{+0.03} \mathrm{mag}$, and $z=3.08_{-0.06}^{+0.11}$ to at least the $3 \sigma$ level (Fig. 3). The best fits for the other dust models return consistent results, and we observe no change larger than $3 \sigma$ in the host dust requirement if $30 \%$ more or less of Galactic dust reddening is used. From here on, any fits requiring redshift are set to the best-fit value for simplicity and the intrinsic host galaxy dust absorption to zero. As there is no strong $2175 \AA$ feature in the SED, we adopt the best-fit SMC-like dust model, which is favoured for the majority of GRB afterglows (e.g., Kann et al. 2010). The LMC and MW-like dust models prefer host galaxy dust quantities of $A_{V} \sim 0.1 \mathrm{mag}$. However, they remain consistent with the best-fit SMC-like dust model values at the $3 \sigma$ level and do not change the results in the rest of the paper.

No spectroscopic redshift has been reported. Ultraviolet detections would help to improve the redshift estimate. However, the upper limits during the afterglow phase from the UltraViolet Optical Telescope (UVOT; Roming et al. 2005) onboard Swift are not constraining. Also, the detections of the afterglow yielded by co-adding over time frames of $\sim 500 \mathrm{~s}$ (Oates \& Siegel 2012) have poor signal-to-noise and fold in the complexity of the light curve. As a result, the UVOT data do not provide tighter constraints on the redshift. At $z \sim 3$, the Ly-limit is within the $u$-band, and given the large errors, the $u$-band detection and UV-limits are thus consistent with the redshift derived from the GROND data.

\subsection{Host galaxy hydrogen column density}

The X-ray light curve behaves like a typical afterglow component at times of $t>T_{0}+1100 \mathrm{~s}$, showing no spectral evolution with a hardness ratio of $1.5 \pm 0.4$. We fit the X-ray data in the time range of $T_{0}+5496 \mathrm{~s}$ to $T_{0}+1.4 \times 10^{6} \mathrm{~s}$ with a power law model and a fixed redshift of $z=3.08$, resulting in a bestfit hydrogen column density of $N_{\mathrm{H}, \mathrm{X}}=2.1 \pm 0.8 \times 10^{22} \mathrm{~cm}^{-2}$

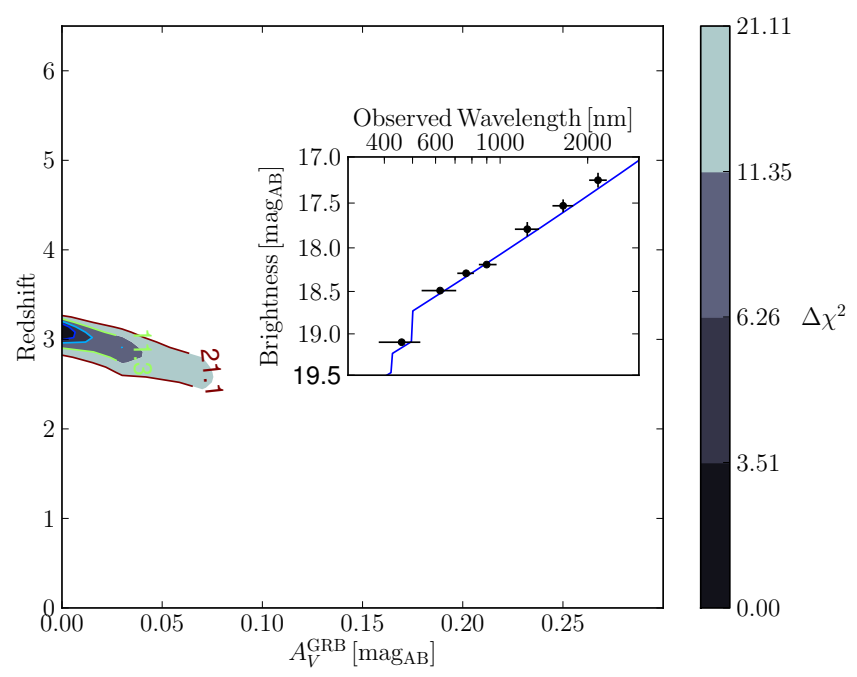

Fig. 3. Contour plot of $\Delta \chi^{2}$ values for each of the host-galaxy dust $\left(A_{V}\right)$ and GRB redshift $(z)$ parameters used in the fitting grid (Sect. 3.1) in comparison to the best-fit power law $(\beta=0.9)$ and SMC-like dust model. The other dust model contour plots are not included, as they do not portray any different information. The inset is the broadband SED, the black dots are GROND data, and the blue line depicts the best-fit power law. The significance levels for 3 d.o.f. are: $1 \sigma=68.3 \%$, $\Delta \chi^{2}=3.5,2 \sigma=90 \%, \Delta \chi^{2}=6.3,3 \sigma=99 \%, \Delta \chi^{2}=11.4$, $4 \sigma=99.99 \%$, and $\Delta \chi^{2}=21.1$.

with $\chi^{2} /$ d.o.f. $=116 / 111$. For consistency, we adopt this value throughout the paper. We note the uncertainties are consistent with zero at the $3 \sigma$ level. However, fixing $N_{\mathrm{H}, \mathrm{X}}=0 \mathrm{~cm}^{-2}$ does not change the overall results outlined in this paper.

\subsection{X-ray emission}

We obtain the best fit X-ray light curve from the Swift online catalogue (Evans et al. 2007, 2009), which determines the temporal slopes based on the type of classification that best fits the data, whether it is canonical, one-break, no-breaks or undefined. By ignoring the flaring activity (Willingale et al. 2007) in the X-ray emission that occurs between $T_{0}+200 \mathrm{~s}$ and $T_{0}+5715 \mathrm{~s}$, the canonical afterglow reproduces the $\mathrm{X}$-ray light curve the best (see Fig. 2) with $\chi^{2}=166 / 146$ (a canonical afterglow usually comprises of three power law segments: a fast initial decay of $3<\alpha<5$ followed by a shallow decay $0.5<\alpha<1.0$ and finishing with a slightly steeper decay $1<\alpha<1.5$, Nousek et al. 2006).

Starting at $T_{0}+72 \mathrm{~s}$, the X-ray light curve begins with a decaying power law with a pre-flare temporal slope of $\alpha_{1, \mathrm{X}}=$ $3.14 \pm 0.18$ and a spectral slope of $\beta_{1, \mathrm{X}}=1.11 \pm 0.01$ between $T_{0}+72 \mathrm{~s}$ and $T_{0}+152 \mathrm{~s}$. The steep decay is then followed by an increase in X-ray emission, where the peak flux is simultaneous to the second prompt-emission peak. Both the flaring and second peak are discussed more thoroughly in Sect. 4.4. After the flaring activity, the X-ray returns to a standard decay with a post-flare temporal slope of $\alpha_{2, \mathrm{X}}=0.54_{-0.17}^{+0.05}$ and spectral slope of $\beta_{2, \mathrm{X}}=0.92 \pm 0.06$. Directly after the X-ray peak, the X-ray emission is systematically below the best-fit line. If achromatic, the X-ray light curve could have the same behaviour as the optical light curve, which is also decreasing at this time. However, there is no coverage of the X-ray emission during the optical rebrightening to place any constraint on the shape of the light curve, so it is possible that $\alpha_{2, \mathrm{X}}$ is underestimated. This decay is then followed by a break at $t_{3, \mathrm{~b}}=T_{0}+2.6 \times 10^{4} \mathrm{~s}$, which 


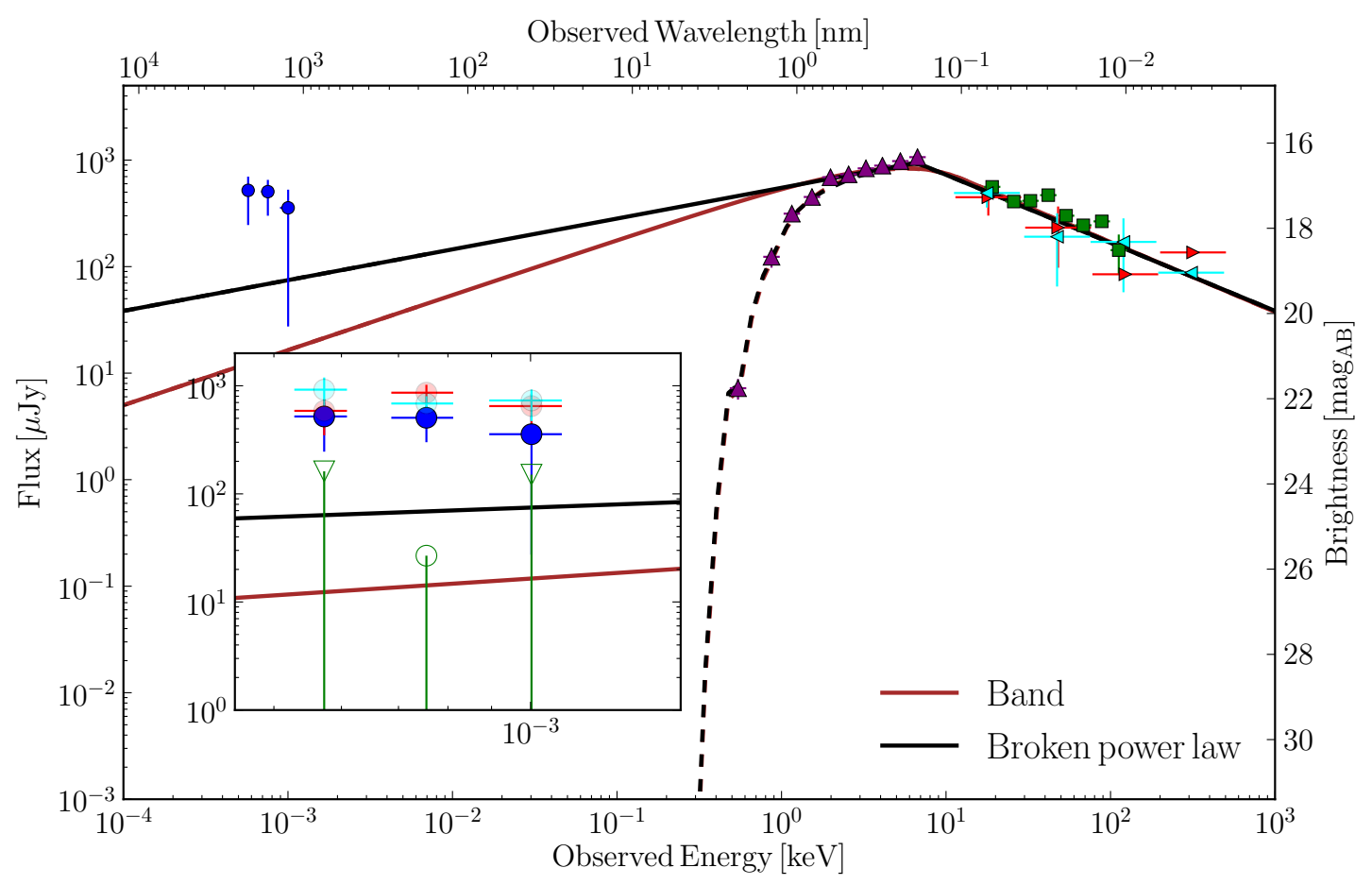

Fig. 4. X-/gamma-ray, NIR SED at the second prompt emission $\left(T_{0}+735 \mathrm{~s}\right.$ ), which is composed of BAT (green squares), XRT (purple upwardtriangles), and GBM's Na and N9 detectors (red rightward-triangles and cyan leftward-triangles, respectively). To make the plot more clear, the data points have been rebinned in energy, and the BGO detectors' non-detections are not included but are consistent with the models. The best-fit models for the broken power law and Band models are depicted as the black and brown lines, respectively. The dashed lines correspond to the best-fit model with the effect of Galactic gas absorption. The inset shows a zoom in of the NIR wavelengths. The open green circle and triangles are with the expected afterglow flux subtracted, which was determined from the best-fit temporal power law. The transparent cyan and red dots denote the fluxes measured at $T_{0}+759$ and $T_{0}+769 \mathrm{~s}$, respectively, which can also be seen in Fig. 5 .

steepens the decay to a final temporal slope of $\alpha_{3, \mathrm{X}}=1.38_{-0.09}^{+0.06}$ and spectral slope $\beta_{3, \mathrm{X}}=0.96 \pm 0.06$.

\subsection{Optical/NIR emission}

The NIR emission depicts no visible synchronous rebrightening during the second prompt emission, and we defer the reader to later discussions (Sect. 4.4). We assume that a second component, which is most likely an afterglow related to the first prompt emission, is dominating the NIR wavelengths. In the external shock model (Sari et al. 1998), the afterglow phase is believed to be when the fireball begins to decelerate as it ploughs into the interstellar medium or progenitor winds, which results in a powerlaw decay (alternative models also require a power-law decay, e.g., the canon ball model; Dar \& de Rújula 2004). Therefore, we fit a double broken power law of the Beuermann et al. (1999) type to the seven bands of GROND simultaneously. The best-fit solution seen in Fig. 2 has $\chi^{2} /$ d.o.f. $=166 / 115$, which is high primarily due to the early and late NIR data. Removing it yields a reduced- $\chi^{2} \sim 1$. This suggests that the NIR uncertainties are being underestimated, but also could be a result of intrinsic variability as a result of flaring activity (see Fig. 5). The NIR (and later optical) emission begins with a shallow decay with a temporal slope of $\alpha_{\mathrm{opt}, 1}=0.15 \pm 0.03$ and then breaks at a time $T_{0}+(750 \pm 19) \mathrm{s}$ to a temporal slope of $\alpha_{\mathrm{opt}, 2}=2.0 \pm 0.1$ with a smoothness $s_{\text {opt } 12}=8.0 \pm 1.5$, which is a steep slope but expected from a reverse shock (Kobayashi \& Zhang 2003). At a time of $\sim T_{0}+1450 \mathrm{~s}$, the optical emission begins to rebrighten with $\alpha_{\text {opt } 3}=-1.8 \pm 0.2$ until it reaches a maximum at $T_{0}+$ $(1669 \pm 10) \mathrm{s}$ with a smoothness of $s_{\mathrm{opt}, 34}=9.8 \pm 0.2$ and, once again, begins to decay with $\alpha_{\mathrm{opt}, 4}=0.59 \pm 0.02$. The spectral slope remains constant throughout with $\beta_{\text {opt }, 34}=0.90 \pm 0.04$. The GROND coverage does not begin again until $T_{0}+8 \times 10^{4} \mathrm{~s}$ but is consistent with a steeper slope of $\alpha_{\text {opt }, 5}=1.19 \pm 0.10$, which is consistent with the X-ray light curve.

\subsection{Broadband prompt-emission spectrum}

We construct a broadband SED at the time of the second prompt emission (Peak 2), which occurs at a mid-time of $T_{0}+$ $(735 \pm 10) \mathrm{s}$. We utilise the three NIR filters of GROND $(\mathrm{JHK})$, Swift/BAT, Swift/XRT, and Fermi/GBM (Fig. 4). As mentioned above, the source was unfortunately located outside the field-ofview of the optical bands of GROND at this time. A common time interval of $10 \mathrm{~s}$ has been used because this is the minimum integration time of the GROND/NIR images that were taken. The spectra from X-ray to gamma-ray wavelengths were fit in XSPEC v12.7.1 with a power law (PL), pow, a broken power law (BPL), bknpow, and the Band function, grbm, and each of the resulting best-fit parameters can be found in Table 2 or are depicted in Fig. 4. We fit the models twice, once including the NIR data and once without it. For fits, which do not include NIR data, we extrapolate each of the models to the NIR wavelengths $(\sim 1 \mu \mathrm{m})$, as seen in the inset of Fig. 4.

\section{Discussion}

\subsection{High latitude emission: deceleration radius and Lorentz factor of the first prompt peak}

We consider the initial steep decay of the X-ray emission (Sect. 3.3), which is usually associated with high latitude 
Table 2. Best-fit parameters for each spectral model for Peak 2.

\begin{tabular}{lcclc}
\hline \hline Model & $\chi^{2} /$ d.o.f. & $f^{a}$ & Parameter & Value \\
\hline $\mathrm{PL}$ & $438 / 342$ & $0.07_{-0.11}^{+0.02}$ & $\beta$ & $0.32 \pm 0.02$ \\
& & & $E_{\text {iso }}^{c}\left[10^{53} \mathrm{erg}\right]$ & 3.3 \\
\hline $\mathrm{PL}^{b}$ & $676 / 345$ & $1.0_{-1.7}^{+0.3}$ & $\beta$ & $0.14 \pm 0.08$ \\
& & & $E_{\text {iso }}^{c}\left[10^{53} \mathrm{erg}\right]$ & 6.2 \\
\hline $\mathrm{BPL}$ & $306 / 340$ & $10_{-3}^{+4}$ & $\beta_{1}$ & $-0.29 \pm 0.06$ \\
& & & $\beta_{2}$ & $0.64 \pm 0.05$ \\
& & & $E_{\mathrm{b}}[\mathrm{keV}]$ & $6.60 \pm 0.85$ \\
& & & $E_{\text {iso }}^{c}\left[10^{53} \mathrm{erg}\right]$ & 1.7 \\
\hline $\mathrm{BPL}^{b}$ & $316 / 343$ & $4_{-2}^{+2}$ & $\beta_{1}$ & $-0.18 \pm 0.05$ \\
& & & $\beta_{2}$ & $0.63 \pm 0.06$ \\
& & & $E_{\mathrm{b}}[\mathrm{keV}]$ & $7.13 \pm 1.14$ \\
& & & $E_{\text {iso }}^{c}\left[10^{53} \mathrm{erg}\right]$ & 1.7 \\
\hline $\mathrm{Band}^{3}$ & $310 / 340$ & $48_{-12}^{+175}$ & $\beta_{1}$ & $-0.48 \pm 0.13$ \\
& & & $\beta_{2}$ & $-1.66 \pm 0.06$ \\
& & & $E_{\mathrm{b}}[\mathrm{keV}]$ & $11 \pm 188$ \\
& & & $E_{\text {iso }}^{c}\left[10^{53} \mathrm{erg}\right]$ & 0.9 \\
\hline Band $^{b}$ & $327 / 343$ & $23_{-7}^{+114}$ & $\beta_{1}$ & $-0.58 \pm 0.11$ \\
& & & $\beta_{2}$ & $-1.65 \pm 0.06$ \\
& & & $E_{\mathrm{b}}[\mathrm{keV}]$ & $14 \pm 8$ \\
& & & $E_{\text {iso }}^{c}\left[10^{53} \mathrm{erg}\right]$ & 0.7 \\
\hline
\end{tabular}

Notes. ${ }^{(a)}$ The ratio of the observed $J$-band flux to the expected $J$-band flux, i.e., $f=J_{\text {obs }} / J_{\text {exp. }}{ }^{(b)}$ Fits that have included the NIR channels. All fits have assumed a redshift of $z=3.08$ and $N_{\mathrm{H}, \mathrm{X}}=2.1 \times 10^{22} \mathrm{~cm}^{-2}$ for simplicity, see Sects. 3.1 and 3.2. ${ }^{(c)}$ Isotropic-equivalent energy calculated over the range of $0.1 \mathrm{keV}$ to $10^{4} \mathrm{keV}$ (see, e.g., Elliott et al. 2012a).

emission (see, e.g., Zhang et al. 2006) and compare the fitted values to the expected closure relations (Kumar \& Panaitescu 2000). We find that $\alpha_{1, \text { closure, }}=2+\beta_{1, \mathrm{X}}=3.11 \pm 0.01$ (cf. $\alpha_{1, \mathrm{X}}=3.14 \pm 0.18$ ) and that this phase therefore is consistent with being related to the prompt emission (i.e., high-latitude emission) and not the afterglow component. We also calculated the closure relations for an afterglow component (e.g., Sari et al. 1998; Racusin et al. 2009) for $p>2$ within a wind/ISM environment for fast/slow cooling and find that they cannot reproduce the temporal slope $\alpha_{1, \mathrm{X}}$ to at least $3 \sigma$. Finally, the decay index is too steep to be a standard reverse ( $\alpha \sim 2$; Kobayashi \& Zhang 2003) or forward shock ( $\alpha \sim 1$; Sari et al. 1998).

Knowledge of the end time of the high-latitude emission allows us to estimate the radius in which the gamma-rays originate and the Lorentz factor of the shell. We place a limit on the radius in which this emission occurs (e.g. Lazzati \& Begelman 2006; Mészáros 2006; Zhang et al. 2006), $R_{\gamma}$, with the following relation:

$t_{\text {tail }} \lesssim(1+z) \frac{R_{\gamma}}{c} \frac{\theta_{\text {jet }}^{2}}{2}$.

Where $c$ is the speed of light, and $\theta_{\text {jet }}$ is the jet half-opening angle. Using the time at which there is a canonical jet break in the X-ray emission at $t_{3, \mathrm{~b}}=T_{0}+2.6 \times 10^{4} \mathrm{~s}$ (Sect. 3.3), the redshift $z=3.08$ (Sect. 3.1), and the isotropic-equivalent energy $E \sim 10^{53} \mathrm{erg}$ (see Table 2 ) from the first peak, we can estimate the opening angle as $\theta_{\text {jet }}=1.6^{\circ}\left(\frac{n_{\gamma}}{0.2}\right)^{\frac{1}{8}}\left(\frac{n_{0}}{0.1 \mathrm{~cm}^{-3}}\right)^{\frac{1}{8}}$ (Frail et al. 2001). Assuming that the gamma-ray efficiency is $n_{\gamma}=0.2$ and the ISM density is $n_{0}=1 \mathrm{~cm}^{-3}$, results in a prompt emission radius of

$R_{\gamma} \gtrsim \frac{2 c t_{\text {tail }}}{(1+z) \theta_{\text {jet }}^{2}}=1.6 \times 10^{15} \mathrm{~cm}$.
This value could be smaller by a factor of a few if the break in the X-ray light curve is not the jet break. The emission from the high-latitude component is much brighter than the onset of the afterglow, which is not seen until the canonical plateau phase begins, which places an upper limit on the time $\left(t_{\mathrm{dec}}\right)$, and, thus, the radius $\left(R_{\mathrm{dec}}\right)$, at which deceleration of the shock begins. Utilising the observation that the tail emission ends at $T_{0}+152 \mathrm{~s}$ (i.e., $\left.t_{\text {tail }}=152 \mathrm{~s}\right)$, we constrain the bulk Lorentz factor, $\Gamma_{0}$, by applying Eq. (6) of Zhang et al. (2006):

$\Gamma_{0} \approx 1328\left[\frac{E_{\gamma, \text { iso, } 52}(1+z)^{3}}{\left(\frac{n_{\gamma}}{0.2}\right)\left(\frac{n_{0}}{1 \mathrm{~cm}^{-3}}\right) t_{\text {peak }}^{3}}\right]^{\frac{1}{8}} \approx 487$,

where we have assumed $t_{\text {peak }}=152 \mathrm{~s}$ and used the same constants as previously noted. We note that the tail emission could last much longer if the plateau phase is not a result of a rising afterglow component, which would reduce the estimate of the Lorentz factor. For example, a time of $1000 \mathrm{~s}$ would halve the Lorentz factor.

After Peak 1, there are no spectral or temporal slopes that satisfy the high latitude emission closure relations to at least $3 \sigma$, and this period is most likely masked by the complexity of the flaring activity.

\subsection{Optical afterglow rebrightening: deceleration radius and Lorentz factor of the second prompt peak}

The optical afterglow-like component that is observed from $T_{0}+1670 \mathrm{~s}$ decays with a temporal slope of $0.59 \pm 0.02$ and a spectral slope of $0.87_{-0.07}^{+0.04}$. These combinations are not consistent with the standard closure relations for either an ISM or wind environment for any of the frequency ranges to the $3 \sigma$ level. If we assume an ISM environment in the slow cooling regime with a frequency located at $v_{\mathrm{m}}<v<v_{\mathrm{c}}$, it would require that $\alpha_{\text {closure }}=\frac{3 \beta_{\text {opt }, 34}}{2}=1.31_{-0.11}^{+0.06}$, which is much steeper than that observed. The shallow decay of the afterglow could be attributed to an injection of energy, which is consistent with the internal shock shells from the X-ray flaring activity that catch up with the primary forward shock. Assuming an injection of the form $E \propto t^{e}$ (Panaitescu et al. 2006), the difference in slopes of $\Delta \alpha=\alpha_{\text {closure }}-\alpha_{\text {opt }, 4}=1.31-0.59=0.72$ must satisfy the relation $\Delta \alpha=e \times 1.36$ in an ISM environment (Panaitescu et al. 2006). Therefore, the flatter slope can be explained with an injection parameter of $e=0.53$.

The rise time of the afterglow component, whether it be the forward or reverse shock, can be used to estimate the bulk Lorentz factor of the ejecta at the deceleration radius. We treat this shell as a thin shell, regardless of whether it is associated with the first or second prompt emission, as there is a clear delay in both cases between the gamma-ray emission and the maximum of the afterglow. Using Eq. (3),

$\Gamma_{0}^{\prime} \approx 247$

where any primed value is related to the second prompt emission. Therefore, the deceleration radius is

$R_{\text {dec }}^{\prime}=2 c \Gamma^{\prime 2} t_{\text {peak }}^{\prime}=3.4 \times 10^{18} \mathrm{~cm}$,

where we have assumed that the afterglow component is a result of the second peak, which occurs at $t_{\text {peak }}^{\prime}=934 \mathrm{~s}$. We have used the same fixed parameters as outlined in the previous section. 


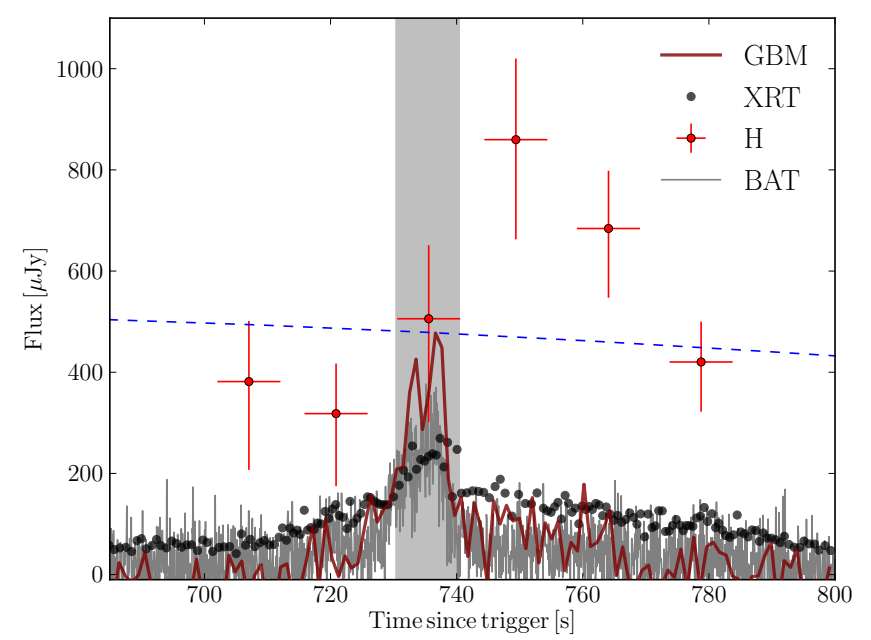

Fig. 5. High time-resolution light curve of the second prompt emission of GRB 121217A, which includes BAT, GBM, XRT, and the $H$-band of GROND. The best-fit $H$-band afterglow light curve from Sect. 3.4 is shown by the dashed blue line.

\subsection{NIR rebrightening during prompt emission}

The high time-resolution light curve seen in Fig. 5 shows that there is a rebrightening in the NIR wavelengths by a factor of $2.7 \pm 0.6$, but this is delayed from the peak of the X-ray/gammaray emission by $(14 \pm 7) \mathrm{s}$. This change in flux is incredibly small when compared to the rebrightening in the X-rays, which changes by a factor of $\sim 100$.

A sample of eighteen bursts from Kopač et al. (2013) that have optical/NIR coverage in a single band during the prompt phase exhibit temporal slopes that are on average $\alpha<-5$. This is in stark contrast to the change in flux of GRB 121217A, which reinforces the idea that the flux from the prompt emission is being outshone by the afterglow emission.

The delay can be explained by considering two different internal-shock components or two shock emissions in a single internal shock component. In a simple internal shock model (the random shell model, e.g., Kobayashi et al. 1997), an internal shock is described by a collision of two shells. Two shocks propagate in the outer and inner shell and if the two shells have very different mass densities the typical frequencies of the emission from the two shocks would be different. They would then peak at different times.

\subsection{Synchrotron radiation model}

The extension of the best-fit power law overpredicts the flux in the NIR wavelengths by a factor $\sim 10$ (Table 2) and has a reduced $-\chi^{2}$ that is more than $3 \sigma$ away from a perfect fit (reduced- $\chi^{2}=1$ ), so we ignore it from here on. Both the broken power law and Band model fits have a reduced- $\chi^{2}$ that is within $3 \sigma$ to a value of 1 . Within synchrotron radiation theory, the break in the power law could exist for two reasons: (i) fast/slow cooling of the electron population or (ii) photon self-absorption by the electron population.

\subsubsection{Fast and slow cooling}

The synchrotron radiation model predicts that the electrons have a spectral slope of $v^{\frac{1}{3}}$ below the maximum injection frequency $v_{\mathrm{m}}$. The slope above this frequency is governed by the rate at which the electrons cool. If they cool faster than the dynamical time of the shock, they have a slope of $v^{-\frac{1}{2}}$, and if they are slower than the dynamical time, then a slope of $v^{-\frac{(p-1)}{2}}$ where $p$ is the electron distribution power law index (Sari et al. 1998).

The best-fit broken power laws (Table 2) below the best-fit break energy have slopes of $\beta_{1}=-0.29 \pm 0.06$ and $\beta_{1}=-0.18 \pm$ 0.05 , respectively, and are consistent with a slope of $v^{\frac{1}{3}}$. Also, the slopes above the break frequency, $\beta_{2}=0.64 \pm 0.05$ and $\beta_{2}=$ $0.63 \pm 0.06$, are still consistent with a slope of $v^{-\frac{1}{2}}$ to the $3 \sigma$ level, assuming fast cooling. In addition, a slope of $\beta=0.64$ would correspond to an electron index of $p=2.3$ for the slow cooling regime, above the break frequency, which is a reasonable value in comparison to theory and observations (e.g., Rossi et al. 2011).

Unfortunately, the broken power law predicts a lower value of the flux expected from the NIR emission. This prediction in combination with the observed small rebrightening in the NIR during the second prompt emission would suggest a secondary component is dominating the emission. The secondary component is most likely afterglow emission related to the first promptemission shell, as we have already noted (for another example, see, e.g., Krühler et al. 2009). We subtract the flux of the bestfit power law to the NIR light curve (see Sect. 3.4 or Fig. 5), which we attribute to an afterglow component during the second prompt-emission and plot the corresponding flux of the GROND $J H K$ bands in Fig. 4. This places the observed spectrum in good agreement with the synchrotron radiation model (or any other model with a similar spectral index).

\subsubsection{Synchrotron self-absorption frequency}

Inclusion of a secondary afterglow component is required to explain the excess flux of the NIR in combination with synchrotron radiation. However, the absorption frequency, $v_{\mathrm{a}}^{\prime}$, may also be between the optical and X-ray frequencies that is hidden underneath the secondary emission component. Self-absorption occurs when the optical wavelength photons are being absorbed by the radiating electrons and this occurs at the self-absorption frequency, $v_{\mathrm{a}}^{\prime}$. We consider the two scenarios outlined in Shen \& Zhang (2009), where the self-absorption frequency is below the optical frequency, $v_{\mathrm{a}}^{\prime}<v_{\mathrm{opt}}^{\prime}<v_{\mathrm{p}}^{\prime}$ (case III of Shen \& Zhang 2009), or the self-absorption frequency is between the optical and X-ray observing frequencies, $v_{\mathrm{opt}}^{\prime}<v_{\mathrm{a}}^{\prime}<v_{\mathrm{X}}^{\prime}$ (case IV of Shen \& Zhang 2009). These frequencies are set by properties of the initial fireball, the emission radius, $R_{\gamma}^{\prime}$, the Lorentz factor, $\Gamma^{\prime}$, and the magnetic field, $B^{\prime}$. There is no visible spectral break in the prompt emission to define $v_{\mathrm{p}}$. At this stage, we can only set an upper limit of $v_{\mathrm{p}}>2.4 \times 10^{20} \mathrm{~Hz}$ with an emission of ${f^{\prime}}_{\nu_{\mathrm{p}}^{\prime}}=40 \mu \mathrm{Jy}$ by assuming the best-fit power law. Using equation 8 from Shen \& Zhang (2009) for case III, this would place a constraint on the self-absorption frequency of

$v_{\mathrm{a}}^{\prime}=\left[\frac{f^{\prime}{v_{\mathrm{p}}^{\prime}}^{\prime}}{f_{\gamma_{\mathrm{opt}}^{\prime}}^{\prime}}\left(\frac{v_{\mathrm{p}}^{\prime}}{v_{\mathrm{m}}^{\prime}}\right)^{\beta} v_{\mathrm{opt}}^{\prime}{ }^{2} v_{\mathrm{m}}^{\prime}-\frac{1}{3}\right]^{\frac{3}{5}}=2.6 \times 10^{14} \mathrm{~Hz}$

where the measured flux in the $H$-band without the afterglow component is ${f^{\prime}}_{v_{\mathrm{opt}}}=27 \mu \mathrm{Jy}, v_{\mathrm{opt}}^{\prime}=1.8 \times 10^{14} \mathrm{~Hz}, v_{\mathrm{m}}^{\prime}=$ $1.6 \times 10^{18} \mathrm{~Hz}$, and $\beta=0.64$ are from the best fit power law. We have also assumed the self-absorption is optically thin (i.e., ${v_{\mathrm{opt}}^{\prime}}^{2}$ ) but the optically thick case is equally as valid (i.e., $v_{\mathrm{opt}}^{\prime}{ }^{\frac{5}{2}}$ ). The determined self-absorption frequency satisfies the relation 


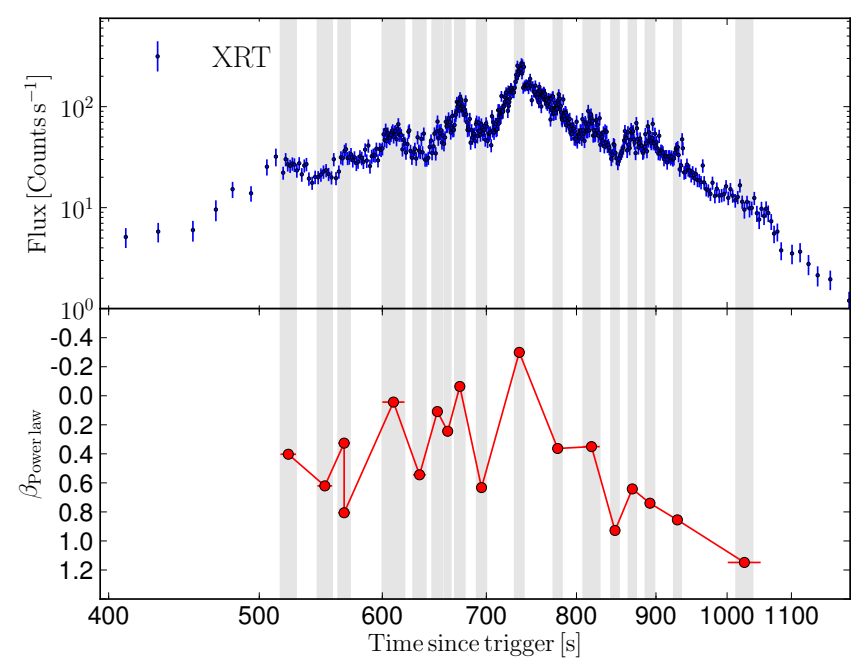

Fig. 6. Top panel: zoom in of the X-ray light curve during the second prompt emission that peaked at $t=T_{0}+735 \mathrm{~s}$. Bottom panel: spectral index of the best-fit power law, $\beta$, for time-sliced spectra shown by the grey shaded bars. The best-fit host gas absorption from the complete prompt SED has been assumed.

$v_{\mathrm{opt}}^{\prime}<v_{\mathrm{a}}^{\prime}<v_{\mathrm{X}}^{\prime}$ for the optically thin absorption but not the optically thick. In the optically, thin case the emission radius can be constrained, using Shen \& Zhang (2009) Eqs. (12) and (A17), to

$R_{\gamma}^{\prime}=4.3 \times 10^{14} \Gamma_{300}^{\prime \frac{3}{4}} B_{5}^{\prime \frac{1}{4}} \mathrm{~cm}$,

using the same values outlined previously. This value is of the order of those determined in other works (e.g., Shen \& Zhang 2009). Substituting the Lorentz factor (Eq. (4)) into Eq. (7) results in an estimate of the required magnetic field of

$B^{\prime}{ }_{5} \sim 1.2 R_{\gamma, 14}^{\prime 4} \mathrm{G}$

\subsection{Flaring activity}

The rebrightening period of the X-ray emission around the second gamma-ray peak is shown in Fig. 6 during which several pulses and dips are evident. It has a maximum peak at the same time as the prompt emission. As already noted, there is an associated gamma-ray pulse for each of the main pulses in the X-ray emission. The length of activity at X-ray wavelengths is $t \sim 600 \mathrm{~s}$, whereas each of the prompt pulses lasts for $t \sim 10-50 \mathrm{~s}$, which is at least twenty times shorter (see also Fig. 2). We fit a power law for the times at which the X-ray light curve shows bumpy features to obtain the spectral slopes.

The power law spectral index begins at $\beta \sim 0.4$ and then approaches very flat values during each of the bumpy features. It is the most flat at the time the prompt emission occurs after which it becomes spectrally soft again, settling at $\beta \sim 1.0$. Each of these bumps could be the result of slow shells (of low Lorentz Factors), causing internal shocks.

In addition, we fit the other prompt emission peaks, 3a, 3b, $3 \mathrm{c}, 3 \mathrm{~d}$, and $3 \mathrm{e}$ with broken power laws and Band functions (see Fig. 1) and plot them alongside the best-fit spectrum of the second prompt emission in Fig. 7. The spectra for which the frequency break or peak energy cannot be constrained are only plotted up to $0.1 \mathrm{keV}$. As already noted, we can think of each bump as being an internal shock of fireballs with varying Lorentz factors. Each peak is then associated with a different

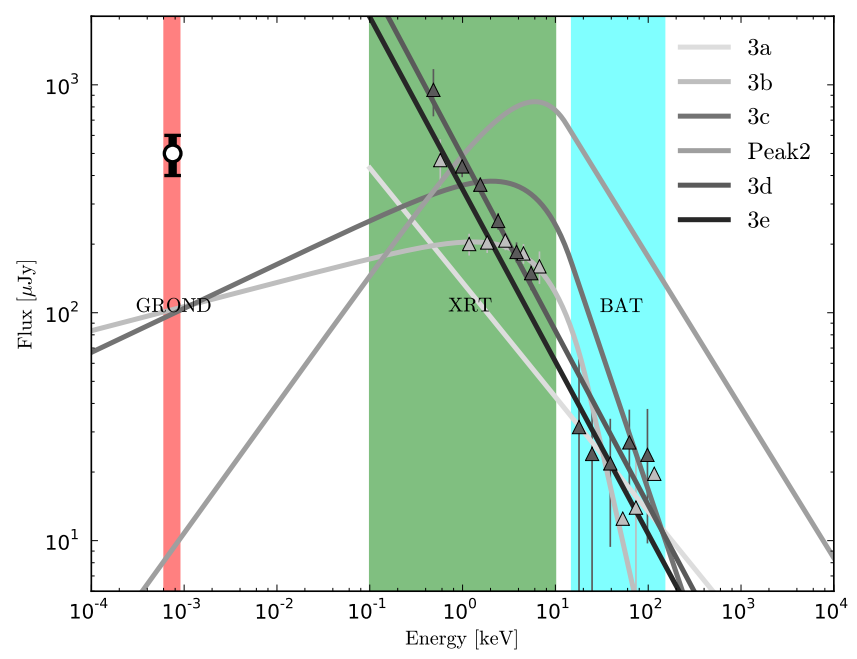

Fig. 7. Band functions for the six prompt emission peaks. Each coloured column shows the wavelength coverage of the instruments (We neglect GBM for clarity as it covers parts of the XRT and BAT.). They show that as the peak energy moves to lower energies the flux in the gamma-ray wavelengths decreases substantially in comparison to the higher peak energy. It can be seen that a higher flux in gamma-rays corresponds to a lower flux in the optical/NIR regime. No constraint on the peak energy was possible for $3 \mathrm{a}, 3 \mathrm{~d}$, and $3 \mathrm{e}$, because they could lie anywhere below $0.1 \mathrm{keV}$, so were refit with a power law. The open circle depicts the (unsubtracted) $J$-band flux measured with GROND during Peak 2. The filled triangles are the X-ray and BAT gamma-ray data, and for reasons of clarity, we only show them for peaks $3 \mathrm{~b}$ and $3 \mathrm{~d}$. The data has been corrected for the Galactic and host hydrogen absorption from the bestfit models. The best-fit parameters and goodness-of-fit for each of the peaks can be found in Table 3 .

maximum injection frequency $v_{\mathrm{m}}$. The larger the $v_{\mathrm{m}}$ of the shock, the brighter it is in the gamma-rays (neglecting any changes of spectral slope). The brightest is detectable by the gamma-ray telescopes. The lower $v_{\mathrm{m}}$ is, the dimmer the gamma-ray emission is (i.e., peaks 3a-e). It is possibly not detected at all, while still remaining bright at X-ray wavelengths (i.e., peaks that are seen in the X-ray emission but not in the gamma-rays). For example, the brightest X-ray and gamma-ray emission is Peak 2, which also has the largest injection frequency. However, we note that is also possible to explain all the features with a simple Band-like spectrum where the injection frequency described previously would be replaced by the peak energy $\left(E_{\text {peak }}\right)$ that is associated with every flare that occurs.

\section{Conclusions}

The Swift/Fermi burst GRB 121217A was observed with two satellites and one ground-based telescope with five different instruments covering the NIR, X-ray, and gamma-ray wavelengths during a secondary prompt-emission period. The NIR emission exhibits a rebrightening during the prompt episode, which is much smaller in comparison to cases, such as the Naked-Eye Burst (080319B). However, the X-ray emission increases by a factor of a hundred and exhibits several X-ray/gamma-ray flares.

The X-ray/gamma-ray spectrum of the second prompt emission is well described by a broken power law but underpredicts the flux expected in the NIR wavelengths. Attributing the additional emission to an afterglow component associated with the first prompt emission and subtracting its contribution would give a flux consistent with an extrapolation of the prompt spectrum 
Table 3. Best-fit parameters for each peak.

\begin{tabular}{lcclc}
\hline \hline Name & Model & $\chi^{2} /$ d.o.f. & Parameter & Value \\
\hline 3a & PL $^{a}$ & $91 / 88$ & $\beta$ & $0.50 \pm 0.06$ \\
\hline 3b & Band & $58 / 77$ & $\beta_{1}$ & $-0.90 \pm 0.20$ \\
& & & $\beta_{2}$ & $-2.82 \pm 1.10$ \\
& & & $E_{\mathrm{b}}[\mathrm{keV}]$ & $11 \pm 33$ \\
\hline 3c & \multirow{2}{*}{ Band } & \multirow{2}{*}{$147 / 98$} & $\beta_{1}$ & $-0.81 \pm 0.14$ \\
& & & $\beta_{2}$ & $-2.20 \pm 0.26$ \\
& & & $E_{\mathrm{b}}[\mathrm{keV}]$ & $11 \pm 240$ \\
\hline 3d & $\mathrm{PL}$ & $81 / 85$ & $\beta$ & $0.76 \pm 0.06$ \\
\hline 3e & $\mathrm{PL}$ & $81 / 78$ & $\beta$ & $0.76 \pm 0.07$
\end{tabular}

Notes. ${ }^{(a)}$ When the Band model returned two slopes of the same value, the data were refit using a power-law model.

of the GRB. In terms of a synchrotron radiation model, the break frequency is interpreted as the maximum injection frequency, $v_{\mathrm{m}}$, at an energy of $E_{\mathrm{m}}=6.6 \mathrm{keV}$. The possibility of the self-absorption frequency existing between the X-ray and optical frequencies allows the radius to be constrained to $R_{\gamma}^{\prime}=$ $4.3 \times 10^{14} \Gamma_{300}^{\frac{3}{4}} B_{5}^{\prime \frac{1}{4}} \mathrm{~cm}$. Estimates of the bulk Lorentz factor are obtained from the peak of the afterglow emission of $\Gamma_{0} \sim 300$. By assuming a standard emission radius in an internal shock model of $R \sim 10^{14} \mathrm{~cm}$, it would suggest a magnetic field strength of $B \sim 10^{5} \mathrm{G}$. A photometric redshift of $z=3.1 \pm 0.1$ is determined from the afterglow emission.

Finally, the X-ray emission has several flaring episodes both before and after the prompt emission, showing that the central engine is active even after the initial prompt emission. The flaring can be explained in terms of the internal shock model as the collision of several fireball shells (with varying Lorentz factors) that are then easily described by synchrotron radiation theory. The lack of any large variability in the NIR wavelengths is then a result of a combination of the (i) dominating afterglow component; (ii) the synchrotron cooling slope of $v^{\frac{1}{3}}$; and (iii) possible self-absorption by the electron population.

We presented GRB 121217A, which was observed simultaneously in multiple NIR filters and by X-ray and gammaray telescopes during its prompt emission. We show that it can be explained in the framework of the internal shock model. Further observations that have high-time resolution $(\sim 10 \mathrm{~s})$ with high signal-to-noise in the optical/NIR wavelengths, which are achievable at such facilities as the Very Large Telescope, in combination with other space-bound facilities that allow a wavelength coverage of $10^{-3} \mathrm{keV}$ to $10^{3} \mathrm{keV}$ are required to further distinguish between the underlying prompt-emission mechanism. These include the internal shock model, magnetic dissipation models in a Poynting-flux-dominated flow, or a dissipative photosphere model.

Acknowledgements. We thank the anonymous referee for their constructive comments. We thank A. Beloborodov, Z. Bosnjak, R. Mochkovitch, S. Xiong, and B. B. Zhang for their comments and suggestions. Part of the funding for GROND (both hardware as well as personnel) was generously granted from the Leibniz-Prize to Prof. G. Hasinger (DFG grant HA 1850/28-1). This work made use of data supplied by the UK Swift Science Data Centre at the University of Leicester. We thank Taka Sakamoto and Scott D. Barthelmy for the public BAT data. H.F.Y. acknowledges support by the DFG cluster of excellence "Origin and Structure of the Universe". S.S. acknowledges support by the Thüringer Ministerium für Bildung, Wissenschaft und Kultur under FKZ 12010-514. P.S. acknowledges support by DFG grant SA 2001/1-1. P.S. and
M.T. acknowledge support through the Sofja Kovalevskaja Award from the Alexander von Humboldt Foundation of Germany. S.K.l. and A.G.N. acknowledge support by DFG grant K1 766/16-1. A.R., A.G.N., and A.K. are grateful for travel funding support through MPE. A.R. acknowledges support by the Thüringer Landessternwarte Tautenburg. K.V. acknowledges support by DFG grant SA 2001/2-1. Swift is supported at PSU by NASA grant NAS5-00136. SKo acknowledges support from the STFC. MS is supported by NASA contract NAS5-00136. T.K. acknowledges support by the European Commission under the Marie Curie Intra-European Fellowship Programme in FP7. The Dark Cosmology Centre is funded by the Danish National Research Foundation.

\section{References}

Aihara, H., Allende Prieto, C., An, D., et al. 2011, ApJS, 193, 29

Akerlof, C., Balsano, R., Barthelmy, S., et al. 1999, Nature, 398, 400

Atwood, W. B., Abdo, A. A., Ackermann, M., et al. 2009, ApJ, 697, 1071

Barthelmy, S. D., Barbier, L. M., Cummings, J. R., et al. 2005, Space Sci. Rev., 120,143

Beskin, G., Karpov, S., Bondar, S., et al. 2010, ApJ, 719, L10

Beuermann, K., Hessman, F. V., Reinsch, K., et al. 1999, A\&A, 352, L26

Blake, C. H., Bloom, J. S., Starr, D. L., et al. 2005, Nature, 435, 181

Bloom, J. S., Perley, D. A., Li, W., et al. 2009, ApJ, 691, 723

Burrows, D. N., Hill, J. E., Nousek, J. A., et al. 2005, Space Sci. Rev., 120, 165

Cucchiara, A., Cenko, S. B., Bloom, J. S., et al. 2011, ApJ, 743, 154

Cummings, J. R., Sakamoto, T., Barthelmy, S. D., et al. 2012, GRB Coordinates

Network, 14096, 1

Daigne, F., \& Mochkovitch, R. 1998, MNRAS, 296, 275

Dar, A., \& de Rújula, A. 2004, Phys. Rep., 405, 203

Eichler, D., Livio, M., Piran, T., \& Schramm, D. N. 1989, Nature, 340, 126

Elliott, J., Greiner, J., Khochfar, S., et al. 2012a, A\&A, 539, A113

Elliott, J., Schmidl, S., \& Greiner, J. 2012b, GRB Coordinates Network, 14091, 1

Evans, P. A., Beardmore, A. P., Page, K. L., et al. 2007, A\&A, 469, 379

Evans, P. A., Beardmore, A. P., Page, K. L., et al. 2009, MNRAS, 397, 1177

Evans, P. A., Goad, M. R., Osborne, J. P., \& Beardmore, A. P. 2012, GRB

Coordinates Network, 14090, 1

Fermi, E. 1949, Phys. Rev., 75, 1169

Fishman, G. J., Meegan, C. A., Wilson, R. B., et al. 1989, in Bull. Am. Astron. Soc., 21, 860

Frail, D. A., Kulkarni, S. R., Sari, R., et al. 2001, ApJ, 562, L55

Freedman, W. L., \& Madore, B. F. 2010, ARA\&A, 48, 673

Gehrels, N., Chincarini, G., Giommi, P., et al. 2004, ApJ, 611, 1005

Gendre, B., Atteia, J. L., Boër, M., et al. 2012, ApJ, 748, 59

Greiner, J., Bornemann, W., Clemens, C., et al. 2008, PASP, 120, 405

Gruber, D., Krühler, T., Foley, S., et al. 2011, A\&A, 528, A15

Kalberla, P. M. W., Burton, W. B., Hartmann, D., et al. 2005, A\&A, 440, 775

Kann, D. A., Klose, S., Zhang, B., et al. 2010, ApJ, 720, 1513

Klebesadel, R. W., Strong, I. B., \& Olson, R. A. 1973, ApJ, 182, L85

Kobayashi, S., \& Zhang, B. 2003, ApJ, 582, L75

Kobayashi, S., Piran, T., \& Sari, R. 1997, ApJ, 490, 92

Kopač, D., Kobayashi, S., Gomboc, A., et al. 2013, ApJ, 772, 73

Krühler, T., Küpcü Yoldaş, A., Greiner, J., et al. 2008, ApJ, 685, 376

Krühler, T., Greiner, J., McBreen, S., et al. 2009, ApJ, 697, 758

Krühler, T., Schady, P., Greiner, J., et al. 2011, A\&A, 526, A153

Kumar, P. 1999, ApJ, 523, L113

Kumar, P., \& Panaitescu, A. 2000, ApJ, 541, L51

Lazzati, D., \& Begelman, M. C. 2006, ApJ, 641, 972

MacFadyen, A. I., \& Woosley, S. E. 1999, ApJ, 524, 262

Mészáros, P. 2002, ARA\&A, 40, 137

Mészáros, P. 2006, Rep. Prog. Phys., 69, 2259

Narayan, R., Paczynski, B., \& Piran, T. 1992, ApJ, 395, L83

Nousek, J. A., Kouveliotou, C., Grupe, D., et al. 2006, ApJ, 642, 389

Oates, S. R., \& Siegel, M. H. 2012, GRB Coordinates Network, 14092, 1

Paczynski, B. 1998, ApJ, 494, L45

Page, K. L., Willingale, R., Osborne, J. P., et al. 2007, ApJ, 663, 1125

Panaitescu, A., Spada, M., \& Mészáros, P. 1999, ApJ, 522, L105

Panaitescu, A., Mészáros, P., Gehrels, N., Burrows, D., \& Nousek, J. 2006, MNRAS, 366, 1357

Racusin, J. L., Karpov, S. V., Sokolowski, M., et al. 2008, Nature, 455, 183

Racusin, J. L., Liang, E. W., Burrows, D. N., et al. 2009, ApJ, 698, 43

Rees, M. J., \& Meszaros, P. 1994, ApJ, 430, L93

Rees, M. J., \& Mészáros, P. 2005, ApJ, 628, 847

Roming, P. W. A., Kennedy, T. E., Mason, K. O., et al. 2005, Space Sci. Rev., 120,95

Rossi, A., Schulze, S., Klose, S., et al. 2011, A\&A, 529, A142

Sari, R., Piran, T., \& Narayan, R. 1998, ApJ, 497, L17 
Schlafly, E. F., \& Finkbeiner, D. P. 2011, ApJ, 737, 103

Shen, R.-F., \& Zhang, B. 2009, MNRAS, 398, 1936

Siegel, M. H., Barlow, B. N., Burrows, D. N., et al. 2012, GRB Coordinates Network, 14089,

Sironi, L., \& Spitkovsky, A. 2009, ApJ, 698, 1523

Skrutskie, M. F., Cutri, R. M., Stiening, R., et al. 2006, AJ, 131, 1163

Tody, D. 1993, in Astronomical Data Analysis Software and Systems II, eds.

R. J. Hanisch, R. J. V. Brissenden, \& J. Barnes, ASP Conf. Ser., 52, 173

Vestrand, W. T., Wozniak, P. R., Wren, J. A., et al. 2005, Nature, 435, 178

Vestrand, W. T., Wren, J. A., Wozniak, P. R., et al. 2006, Nature, 442, 172

Virgili, F. J., Mundell, C. G., Pal'shin, V., et al. 2013, ApJ, 778, 54

Vurm, I., Beloborodov, A. M., \& Poutanen, J. 2011, ApJ, 738, 77

Willingale, R., O'Brien, P. T., Osborne, J. P., et al. 2007, ApJ, 662, 1093
Woosley, S. E. 1993, ApJ, 405, 273

Wren, J., Vestrand, W. T., Wozniak, P., \& Davis, H. 2013, GRB Coordinates Network, 14476, 1

Yoldaş, A. K., Krühler, T., Greiner, J., et al. 2008, in AIP Conf. Ser. 1000, eds. M. Galassi, D. Palmer, \& E. Fenimore, 227

Yu, D., \& Gruber, D. 2012, GRB Coordinates Network, 14094, 1

Zhang, B. 2011, Comptes Rendus Physique, 12, 206

Zhang, B. 2012, in AIP Conf. Ser. 1505, eds. F. A. Aharonian, W. Hofmann, \& F. M. Rieger, 88

Zhang, B., \& Pe'er, A. 2009, ApJ, 700, L65

Zhang, B., \& Yan, H. 2011, ApJ, 726, 90

Zhang, B., Fan, Y. Z., Dyks, J., et al. 2006, ApJ, 642, 354

Zheng, W., Shen, R. F., Sakamoto, T., et al. 2012, ApJ, 751, 90 
J. Elliott et al.: Prompt emission of GRB 121217A from gamma-rays to the near-infrared

\section{Appendix A: Light curve tables}

Table A.1. Optical reference stars.

\begin{tabular}{lccccc}
\hline \hline $\begin{array}{l}\text { RA } \\
(\mathrm{J} 2000)\end{array}$ & $\begin{array}{c}\text { Dec } \\
(\mathrm{J} 2000)\end{array}$ & $\begin{array}{c}g^{\prime} \\
\mathrm{mag}_{\mathrm{AB}}\end{array}$ & $\begin{array}{c}r^{\prime} \\
\mathrm{mag}_{\mathrm{AB}}\end{array}$ & $\begin{array}{c}i^{\prime} \\
\mathrm{mag}_{\mathrm{AB}}\end{array}$ & $\begin{array}{c}z^{\prime} \\
\mathrm{mag}_{\mathrm{AB}}\end{array}$ \\
\hline $10: 14: 45.18$ & $-62: 21: 13.9$ & $18.84 \pm 0.01$ & $18.43 \pm 0.01$ & $18.30 \pm 0.02$ & $18.22 \pm 0.02$ \\
$10: 14: 48.66$ & $-62: 20: 20.5$ & $16.77 \pm 0.01$ & $16.22 \pm 0.01$ & $16.06 \pm 0.01$ & $15.94 \pm 0.01$ \\
$10: 14: 49.68$ & $-62: 20: 29.4$ & $17.29 \pm 0.01$ & $16.16 \pm 0.01$ & $15.78 \pm 0.01$ & $15.51 \pm 0.01$ \\
$10: 14: 57.84$ & $-62: 21: 22.5$ & $17.94 \pm 0.01$ & $17.40 \pm 0.01$ & $17.24 \pm 0.01$ & $17.11 \pm 0.01$ \\
$10: 15: 03.31$ & $-62: 20: 42.4$ & $17.82 \pm 0.01$ & $17.14 \pm 0.01$ & $16.94 \pm 0.01$ & $16.82 \pm 0.01$ \\
\hline
\end{tabular}

Table A.2. GROND photometric data $g^{\prime} r^{\prime} i^{\prime} z^{\prime}$.

\begin{tabular}{llcccc}
\hline \hline$T_{\text {mid }}-T_{0}$ & Exposure & $\begin{array}{c}g^{\prime} \\
\operatorname{mag}_{\mathrm{AB}}\end{array}$ & $\begin{array}{c}r^{\prime} \\
\mathrm{mag}_{\mathrm{AB}}\end{array}$ & $\begin{array}{c}i^{\prime} \\
\mathrm{mag}_{\mathrm{AB}}\end{array}$ & $\begin{array}{c}z^{\prime} \\
\mathrm{mag}_{\mathrm{AB}}\end{array}$ \\
\hline 1120 & $\mathrm{~s}$ & $20.41 \pm 0.04$ & $19.49 \pm 0.04$ & $19.12 \pm 0.04$ & $18.83 \pm 0.04$ \\
1229 & 33 & $20.52 \pm 0.06$ & $19.49 \pm 0.05$ & $19.16 \pm 0.06$ & $18.79 \pm 0.05$ \\
1338 & 33 & $20.59 \pm 0.04$ & $19.66 \pm 0.04$ & $19.17 \pm 0.04$ & $18.99 \pm 0.05$ \\
1447 & 33 & $20.69 \pm 0.07$ & $19.59 \pm 0.04$ & $19.20 \pm 0.05$ & $18.94 \pm 0.05$ \\
1592 & 58 & $20.11 \pm 0.04$ & $19.16 \pm 0.02$ & $18.78 \pm 0.03$ & $18.48 \pm 0.04$ \\
1787 & 58 & $19.99 \pm 0.03$ & $18.96 \pm 0.01$ & $18.59 \pm 0.03$ & $18.28 \pm 0.03$ \\
1981 & 58 & $19.94 \pm 0.02$ & $19.00 \pm 0.02$ & $18.59 \pm 0.03$ & $18.33 \pm 0.03$ \\
2173 & 58 & $19.96 \pm 0.04$ & $19.02 \pm 0.02$ & $18.61 \pm 0.03$ & $18.39 \pm 0.04$ \\
2374 & 58 & $20.06 \pm 0.04$ & $19.08 \pm 0.02$ & $18.69 \pm 0.03$ & $18.40 \pm 0.03$ \\
2568 & 58 & $20.13 \pm 0.04$ & $19.16 \pm 0.02$ & $18.76 \pm 0.03$ & $18.52 \pm 0.03$ \\
2760 & 58 & $20.21 \pm 0.03$ & $19.23 \pm 0.02$ & $18.85 \pm 0.03$ & $18.55 \pm 0.03$ \\
2953 & 58 & $20.20 \pm 0.03$ & $19.27 \pm 0.02$ & $18.89 \pm 0.03$ & $18.63 \pm 0.03$ \\
3154 & 58 & $20.28 \pm 0.03$ & $19.29 \pm 0.02$ & $18.90 \pm 0.04$ & $18.66 \pm 0.03$ \\
3346 & 58 & $20.30 \pm 0.03$ & $19.36 \pm 0.02$ & $18.99 \pm 0.03$ & $18.69 \pm 0.03$ \\
3539 & 58 & $20.38 \pm 0.03$ & $19.44 \pm 0.02$ & $19.02 \pm 0.03$ & $18.78 \pm 0.03$ \\
3737 & 58 & $20.43 \pm 0.04$ & $19.46 \pm 0.02$ & $19.12 \pm 0.03$ & $18.83 \pm 0.03$ \\
3940 & 58 & $20.44 \pm 0.05$ & $19.45 \pm 0.03$ & $19.08 \pm 0.04$ & $18.84 \pm 0.04$ \\
4126 & 58 & $20.48 \pm 0.05$ & $19.55 \pm 0.03$ & $19.13 \pm 0.04$ & $18.91 \pm 0.04$ \\
4320 & 58 & $20.54 \pm 0.07$ & $19.58 \pm 0.02$ & $19.16 \pm 0.03$ & $18.92 \pm 0.04$ \\
4518 & 58 & $20.63 \pm 0.07$ & $19.62 \pm 0.03$ & $19.28 \pm 0.03$ & $19.03 \pm 0.03$ \\
4736 & 18 & $20.68 \pm 0.15$ & $19.71 \pm 0.05$ & $19.35 \pm 0.06$ & $18.98 \pm 0.07$ \\
4736 & 18 & $20.73 \pm 0.11$ & $19.70 \pm 0.04$ & $19.35 \pm 0.05$ & $19.08 \pm 0.06$ \\
74739 & 865 & $22.93 \pm 0.08$ & $21.95 \pm 0.05$ & $21.59 \pm 0.06$ & $21.51 \pm 0.09$ \\
86363 & 865 & $23.20 \pm 0.12$ & $21.95 \pm 0.05$ & $21.68 \pm 0.06$ & $21.68 \pm 0.10$ \\
88178 & 870 & $23.21 \pm 0.09$ & $22.14 \pm 0.04$ & $21.80 \pm 0.07$ & $21.64 \pm 0.08$ \\
173620 & 1549 & $>24.46$ & $22.82 \pm 0.09$ & $22.30 \pm 0.13$ & $22.61 \pm 0.19$ \\
347165 & 2688 & $>24.93$ & $23.52 \pm 0.12$ & $23.42 \pm 0.18$ & $23.65 \pm 0.30$ \\
1377575 & 2666 & $>24.09$ & $>24.07$ & $>23.69$ & $>23.57$ \\
1814887 & 4019 & $>25.19$ & $>24.90$ & $>24.16$ & $>23.86$ \\
\hline & & & & &
\end{tabular}

Notes. All magnitudes have not been corrected for Galactic foreground reddening. 
Table A.3. GROND photometric data $J H K_{\mathrm{s}}$.

\begin{tabular}{llccc}
\hline \hline $\begin{array}{l}T_{\text {mid }}-T_{0} \\
\mathrm{~s}\end{array}$ & $\begin{array}{l}\text { Exposure } \\
\mathrm{s}\end{array}$ & $\begin{array}{c}J \\
\mathrm{mag}_{\mathrm{AB}}\end{array}$ & $\begin{array}{c}H \\
\mathrm{mag}_{\mathrm{AB}}\end{array}$ & $\begin{array}{c}K_{s} \\
\mathrm{mag}_{\mathrm{AB}}\end{array}$ \\
\hline 301 & 41 & $17.02 \pm 0.08$ & $17.21 \pm 0.11$ & $16.85 \pm 0.12$ \\
401 & 41 & $17.47 \pm 0.12$ & $17.26 \pm 0.13$ & $16.85 \pm 0.13$ \\
509 & 41 & $17.37 \pm 0.12$ & $17.21 \pm 0.16$ & $16.70 \pm 0.11$ \\
619 & 41 & $17.52 \pm 0.13$ & $17.04 \pm 0.09$ & $17.14 \pm 0.14$ \\
744 & 41 & $17.40 \pm 0.09$ & $16.98 \pm 0.06$ & $16.95 \pm 0.10$ \\
843 & 41 & $17.76 \pm 0.10$ & $17.53 \pm 0.08$ & $17.06 \pm 0.10$ \\
927 & 19 & $17.99 \pm 0.17$ & $17.70 \pm 0.15$ & $17.74 \pm 0.28$ \\
1291 & 205 & $18.36 \pm 0.09$ & $17.92 \pm 0.06$ & $17.89 \pm 0.12$ \\
1909 & 374 & $17.75 \pm 0.04$ & $17.51 \pm 0.04$ & $17.23 \pm 0.06$ \\
2689 & 373 & $17.96 \pm 0.05$ & $17.64 \pm 0.04$ & $17.55 \pm 0.07$ \\
3470 & 375 & $18.20 \pm 0.06$ & $17.95 \pm 0.05$ & $17.87 \pm 0.10$ \\
4254 & 372 & $18.30 \pm 0.08$ & $17.95 \pm 0.06$ & $17.95 \pm 0.10$ \\
4927 & 238 & $18.49 \pm 0.13$ & $18.26 \pm 0.09$ & $18.10 \pm 0.10$ \\
5732 & 331 & $18.48 \pm 0.08$ & $18.46 \pm 0.08$ & $18.41 \pm 0.15$ \\
6577 & 329 & $18.46 \pm 0.14$ & $18.40 \pm 0.08$ & $18.70 \pm 0.16$ \\
7181 & 190 & $\ldots$ & $18.43 \pm 0.23$ & $18.98 \pm 0.28$ \\
83117 & 7317 & $>20.97$ & $>20.52$ & $>20.14$ \\
173757 & 1360 & $>20.77$ & $>20.49$ & $>20.15$ \\
347192 & 2423 & $>20.99$ & $>20.72$ & $>20.28$ \\
1377597 & 2252 & $>21.00$ & $>20.86$ & $>20.40$ \\
1814914 & 3607 & $>21.11$ & $>20.84$ & $>20.55$ \\
\hline
\end{tabular}

Notes. All magnitudes have not been corrected for Galactic foreground reddening.

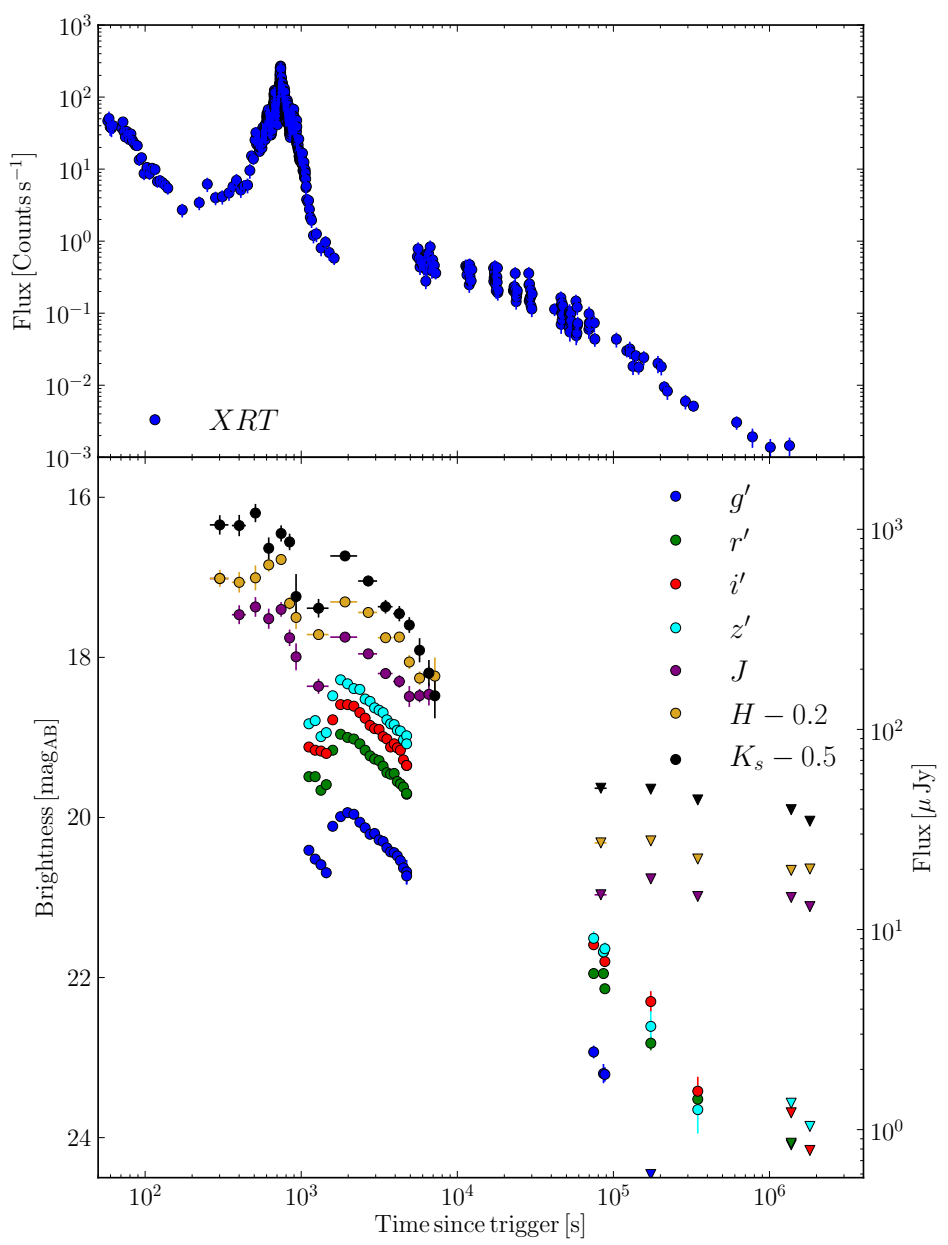

Fig. A.1. Complete GROND and XRT light curves. 